\title{
Elastic Stabilization of a Single-Domain Ferroelectric State in Nanoscale Capacitors and Tunnel Junctions
}

\author{
N. A. Pertsev ${ }^{1,2}$ and H. Kohlstedt ${ }^{1}$ \\ ${ }^{1}$ Institut für Festkörperforschung, Forschungszentrum Jülich, D-52425 Jülich, Germany \\ ${ }^{2}$ A. F. Ioffe Physico-Technical Institute, Russian Academy of Sciences, 194021 St. Petersburg, Russia
}

(Received 14 November 2006; published 22 June 2007)

\begin{abstract}
Taking into account the electrostrictive coupling between inhomogeneous polarization fluctuations and lattice strains in ferroelectric films, we show that, in heterostructures involving strained epitaxial films and metal electrodes, the single-domain state may remain stable against the transformation into a polydomain state down to the nanometer scale. This result indicates that the ferroelectric states with opposite remanent polarizations can be stabilized even in nanoscale capacitors and tunnel junctions, which opens the possibility of their application for memory storage.
\end{abstract}

PACS numbers: 77.80.Dj, 77.55.+f

The persistence of ferroelectricity in ultrathin films is an issue of high fundamental and practical interest. In particular, the stability of states with a nonzero net polarization represents a matter of primary importance. Indeed, the presence of remanent out-of-plane polarization is necessary for the memory applications of ferroelectric films in the form of capacitors [1] and tunnel junctions [2-4].

Dependence of the polarization pattern $\mathbf{P}(\mathbf{r})$ on the film thickness may result from both long-range and short-range interactions. The most widely discussed cause of this size effect is the existence of a depolarizing field [5-10]. The phenomenological theory predicted a long time ago that this field differs from zero even in thin films covered by metal electrodes $[6,8]$. The depolarizing-field effect on polarization was invoked to explain the gradual reduction of tetragonality in ultrathin $\mathrm{PbTiO}_{3}$ films [11] and the polarization relaxation observed in $\mathrm{SrRuO}_{3} / \mathrm{BaTiO}_{3} /$ $\mathrm{SrRuO}_{3}$ capacitors [12]. In addition to the long-range depolarizing field, the film polarization may be affected by the intrinsic surface effect associated with dipole-dipole interactions [13] and by short-range interactions between atomic layers adjacent to the ferroelectric-electrode interface $[14,15]$.

According to the predictions of the mean-field theory $[5,7]$ and recent first-principles calculations $[10,14,15]$, the depolarizing-field effect may lead to the complete disappearance of the ferroelectric phase below some critical film thickness. In these studies, however, the film was assumed to remain in a single-domain state, although the depolarizing field $E_{\text {dep }}$ tends to induce the formation of a $180^{\circ}$ domain structure [16]. Since this transformation strongly reduces the magnitude of $E_{\mathrm{dep}}$, it may prevent the ferroelectric to paraelectric phase transition. Thus, the stability of a single-domain film against the appearance of $180^{\circ}$ domains becomes an extremely important issue.

The stability problem can be solved by studying small inhomogeneous perturbations of the uniform polarization state [17]. The existing solutions of this problem [17,18], however, ignore totally the fact that, owing to the electro- strictive coupling, the polarization fluctuations inevitably modify the lattice strains. This effect is expected to play an important role in the stabilization of the single-domain state in both thin-film and bulk ferroelectrics, because lattice strains strongly influence the magnitude and orientation of polarization in epitaxial films [19-21].

In this Letter, we present a rigorous solution of the stability problem for the single-domain state in ferroelectric films and demonstrate that the electrostrictive coupling between polarization and strain may stabilize this state even in few-nanometer-thick epitaxial layers. This prediction differs drastically from the former results [18], which indicated that the polydomain state of ferroelectric films is almost always preferred over the single-domain one.

We focus on thin films of perovskite ferroelectrics grown on a thick cubic substrate inducing compressive in-plane strains in the film. At thicknesses $t$ outside the nanoscale range, such films stabilize in the tetragonal $c$ phase with the polarization $\mathbf{P}$ orthogonal to the substrate $[19,20]$. Neglecting for clarity the surface effects on $\mathbf{P}(\mathbf{r})$ [1315], we assume the film to be homogeneously polarized in the ground state and first determine the spontaneous polarization, which is necessary for the stability analysis. The film polarization can be calculated from the nonlinear equation of state derived by differentiating the film Helmholtz free energy [21] written in terms of polarization components $P_{i}(i=1,2,3)$. For the $c$ phase $\left(P_{1}=P_{2}=0\right.$, $\left.P_{3} \neq 0\right)$ stable at negative misfit strains $S_{m}$, we obtain

$$
2 a_{3}^{*} P_{3}+4 a_{33}^{*} P_{3}^{3}+6 a_{111} P_{3}^{5}+8 a_{1111} P_{3}^{7}+\cdots=E_{3},
$$

where $a_{3}^{*}=a_{1}+2 S_{m}\left(q_{11} c_{12}-q_{12} c_{11}\right) / c_{11}, a_{33}^{*}=a_{11}-$ $q_{11}^{2} /\left(2 c_{11}\right), a_{1}, a_{i j}, a_{i j k}$, and $a_{i j k l}$ are the dielectric stiffness coefficients at constant strain, $q_{l n}$ are the relevant electrostrictive constants, and $c_{l n}$ are the film elastic stiffnesses at constant polarization. The total electric field $E_{3}$ inside the film can be calculated from the voltage drop $V_{a}$ across the metal-ferroelectric-metal (MFM) heterostructure and the continuity condition of the electric displacement 
$\mathbf{D}=\varepsilon_{0} \mathbf{E}+\mathbf{P}$ at the interfaces $\left(\varepsilon_{0}\right.$ is the permittivity of the vacuum) $[6,7]$. The calculation yields [22]

$$
E_{3}=-\frac{P_{3}}{\varepsilon_{0}+c_{i} t}-\frac{c_{i}}{\left(\varepsilon_{0}+c_{i} t\right)} V_{a}
$$

where $c_{i}$ is the total capacitance of the screening space charge in the electrodes [23]. The substitution of Eq. (2) into Eq. (1) shows that the depolarizing field $E_{\mathrm{dep}}=$ $-P_{3} /\left(\varepsilon_{0}+c_{i} t\right)$ formally renormalizes the coefficient $a_{3}^{*}$ of the lowest-order polarization term, transforming it into $a_{3}^{* *}=a_{3}^{*}+1 /\left[2\left(\varepsilon_{0}+c_{i} t\right)\right]$. Using this expression, we can calculate $P_{3}$ and $E_{\text {dep }}$ as a function of the film thickness $t$.

We performed these calculations for $\mathrm{Pb}\left(\mathrm{Zr}_{0.5} \mathrm{Ti}_{0.5}\right) \mathrm{O}_{3}$ (PZT) and $\mathrm{BaTiO}_{3}$ (BT) films grown on $\mathrm{SrTiO}_{3}$, assuming $S_{m}$ to be equal to a thickness-independent value attained in fully strained MFM trilayers. The spontaneous polarization $P_{s}=P_{3}\left(V_{a}=0\right)$ of PZT films was calculated in the $P^{6}$ approximation, while we used the $P^{8}$ approximation [24] to find $P_{s}$ of BT films [25]. Since the capacitance $c_{i}$ affects
$P_{s}$ only via the product $c_{i} t$, the dependences $P_{s}(t)$ corresponding to different electrode materials can be described by one universal curve $P_{s}\left(t_{\text {eff }}\right)$. The effective film thickness $t_{\text {eff }}$ may be defined as $t_{\text {eff }}=\left(c_{i} / c_{1}\right) t$, where $c_{1}=1 \mathrm{~F} / \mathrm{m}^{2}$.

The calculated curves $P_{s}\left(t_{\text {eff }}\right)$ show that the out-of-plane polarization $P_{s}$ vanishes at a critical film thickness $t_{0}$, which was regarded as a size-induced phase transition $[10,26]$. Just above $t_{0} \cong-1 /\left(2 c_{i} a_{3}^{*}\right)$, the film polarization steeply increases with thickness and reaches values comparable to the bulk polarization (see Fig. 1). At $c_{i}=$ $0.444 \mathrm{~F} / \mathrm{m}^{2}$ characteristic of $\mathrm{SrRuO}_{3}$ electrodes [27], the calculation gives $t_{0} \cong 2 \mathrm{~nm}$ for PZT films and $t_{0} \cong$ $2.6 \mathrm{~nm}$ for BT films [28]. Therefore, even nanoscale capacitors and tunnel junctions may have the out-of-plane polarization sufficient for the memory applications.

Now we shall analyze a wavelike perturbation of the uniform polarization state. Since the polarization distribution becomes inhomogeneous, Eq. (1) should be replaced by the Euler equations involving the gradient terms. For ferroelectrics with a cubic paraelectric phase, these equations in our two-dimensional case [29] become

$$
\begin{aligned}
\left(2 a_{1}+4 a_{11} P_{1}^{2}+2 a_{12} P_{3}^{2}+\cdots\right) P_{1}-2 q_{11} S_{11} P_{1}-2 q_{12}\left(S_{22}+S_{33}\right) P_{1}-2 q_{44} S_{13} P_{3}-E_{1} & =g_{11} P_{1,11}+\left(g_{44}+g_{44}^{\prime}\right) P_{1,33} \\
& +\left(g_{12}+g_{44}-g_{44}^{\prime}\right) P_{3,13}, \\
\left(2 a_{1}+4 a_{11} P_{3}^{2}+2 a_{12} P_{1}^{2}+\cdots\right) P_{3}-2 q_{11} S_{33} P_{3}-2 q_{12}\left(S_{11}+S_{22}\right) P_{3}-2 q_{44} S_{13} P_{1}-E_{3}= & \left(g_{12}+g_{44}+g_{44}^{\prime}\right) P_{1,13} \\
& +\left(g_{44}-g_{44}^{\prime}\right) P_{3,11}+g_{11} P_{3,33},
\end{aligned}
$$

where indices after the comma denote differentiation with respect to the coordinates $x_{1}$ and $x_{3}, g_{l n}$ are the coefficients of the gradient terms in the free-energy expansion [30], and $S_{i j}$ are the lattice strains in the film $\left(S_{22}=S_{m}\right)$. The polarization components involved in Eqs. (3) and (4) can be written as $P_{3}=P_{s}+\delta P_{3}\left(x_{3}\right) \exp \left(i k x_{1}\right)$ and $P_{1}=\delta P_{1}\left(x_{3}\right) \exp \left(i k x_{1}\right)$, with $\delta P_{1}, \delta P_{3} \ll P_{s}$. Similar relations $S_{11}=S_{m}+\delta S_{11}\left(x_{3}\right) \exp \left(i k x_{1}\right), S_{33}=\left(q_{11} P_{s}^{2}-2 c_{12} S_{m}\right) / c_{11}+\delta S_{33}\left(x_{3}\right) \exp \left(i k x_{1}\right)$, $S_{13}=\delta S_{13}\left(x_{3}\right) \exp \left(i k x_{1}\right)$, and $\phi=\phi_{0}\left(x_{3}\right)+\delta \phi\left(x_{3}\right) \exp \left(i k x_{1}\right)$ can be introduced for the film strains and the electrostatic potential $\phi$. Using these formulas and retaining only the lowest-order perturbation terms, we obtain

$$
\begin{gathered}
\left(\tilde{\chi}_{11}+g_{11} k^{2}\right) \delta P_{1}-2 q_{44} P_{s} \delta S_{13}+i k \delta \phi=\left(g_{44}+g_{44}^{\prime}\right) \delta P_{1,33}+i k\left(g_{12}+g_{44}-g_{44}^{\prime}\right) \delta P_{3,3}, \\
{\left[\tilde{\chi}_{33}+\left(g_{44}-g_{44}^{\prime}\right) k^{2}\right] \delta P_{3}-2 q_{11} P_{s} \delta S_{33}-2 q_{12} P_{s} \delta S_{11}+\delta \phi_{, 3}=i k\left(g_{12}+g_{44}+g_{44}^{\prime}\right) \delta P_{1,3}+g_{11} \delta P_{3,33},}
\end{gathered}
$$

where $\quad \tilde{\chi}_{11}=2 a_{1}-2\left(q_{11}+q_{12}-2 q_{12} c_{12} / c_{11}\right) S_{m}+$ $2\left(a_{12}-q_{11} q_{12} / c_{11}\right) P_{s}^{2}+2 a_{112} P_{s}^{4}+\cdots \quad$ and $\tilde{\chi}_{33}=$ $2 a_{1}+4\left[q_{11}\left(c_{12} / c_{11}\right)-q_{12}\right] S_{m}+12\left[a_{11}-q_{11}^{2} /\left(6 c_{11}\right)\right] P_{s}^{2}+$ $30 a_{111} P_{s}^{4}+\cdots$.

In addition to Eqs. (5) and (6), four other equations must be satisfied. First, the electrostatic condition $\operatorname{div} \mathbf{D}=0$ holding inside an insulating film provides a relation between the perturbations $\delta \phi, \delta P_{1}$, and $\delta P_{3}$. Second, the strains $S_{i j}$ in the film must obey the classical compatibility condition $e_{i k l} e_{j m n} S_{l n, k m}=0$ ( $e_{i k l}$ is the permutation symbol), which reduces to one nontrivial equation in our case. Third, the equations of mechanical equilibrium $\sigma_{i j, j}=0$ written for the film stresses $\sigma_{i j}$ yield another two relations. In total, we obtain a system of six differential equations for six unknown functions: $\delta P_{1}, \delta P_{3}, \delta \phi, \delta S_{11}, \delta S_{33}$, and $\delta S_{13}$. The analysis shows that here the terms involving a very small factor $\varepsilon_{0}$ may be neglected. Then the discussed system may be reduced to the following two simultaneous equations:

$$
\begin{aligned}
& {\left[\tilde{\chi}_{33}-2 q_{12} \tilde{q} P_{s}^{2}+\left(g_{44}-g_{44}^{\prime}\right) k^{2}\right] k^{2} \delta P_{3}} \\
& -\left[\tilde{\chi}_{11}+2 q_{44} \tilde{q} P_{s}^{2}+2\left(g_{11}-g_{12}-g_{44}\right) k^{2}\right] \delta P_{3,33} \\
& +\left(g_{44}+g_{44}^{\prime}\right) \delta P_{3,3333}-\left(2 q_{12}-c_{11} \tilde{q}\right) P_{s} k^{2} \delta S_{11} \\
& -\left(q_{44}+2 c_{44} \tilde{q}\right) P_{s} \delta S_{11,33}=0, \\
& 4 c_{44} q_{12} P_{s} k^{4} \delta P_{3}+2\left[\left(c_{12}+2 c_{44}\right) q_{11}-c_{11} q_{12}-c_{12} q_{44}\right] \\
& \times P_{s} k^{2} \delta P_{3,33}-2 c_{11} q_{44} P_{s} \delta P_{3,3333}-2 c_{11} c_{44} k^{4} \delta S_{11} \\
& +\left(c_{11}^{2}-c_{12}^{2}-4 c_{12} c_{44}\right) k^{2} \delta S_{11,33}-2 c_{11} c_{44} \delta S_{11,3333}=0,
\end{aligned}
$$

where $\tilde{q}=\left(2 q_{11}-q_{44}\right) /\left(c_{12}+2 c_{44}\right)$. It should be emphasized that Eqs. (7) and (8) differ dramatically from the 



FIG. 1 (color online). Thickness dependence of the out-ofplane polarization and depolarizing field in ultrathin (a) PZT and (b) BT films grown on $\mathrm{SrTiO}_{3}$. The misfit strain is taken to be $-39 \times 10^{-3}$ for PZT films and $-26 \times 10^{-3}$ for BT films; $T=25^{\circ} \mathrm{C}$. The effective film thickness $t_{\text {eff }}$ is defined in the text. The dashed line shows the thickness below which the singledomain state becomes unstable.

differential equation used to describe the stability of a uniform polarization state formerly [17,18,31]. Indeed, owing to the electrostrictive effect, Eq. (7) contains terms depending on the lattice strain and its second derivative, which were overlooked previously. Moreover, additionally, Eq. (8) must be satisfied because the polarization wave creates an elastic wave in a piezoelectric medium.

Since the system (7) and (8) is homogeneous, we may seek the solution in the form of $\delta P_{3}=A \exp \left(\lambda x_{3}\right), \delta S_{11}=$ $B \exp \left(\lambda x_{3}\right)$. This leads to a homogeneous system of two linear algebraic equations in the unknown coefficients $A$ and $B$. Calculating the determinant $\Delta_{\lambda}$ of this system and setting it to zero, we obtain the characteristic equation $\Delta_{\lambda}(\lambda)=0$ in the form of a quartic algebraic equation with respect to $\lambda^{2}$. When all roots $\lambda_{n}(k)$ of this equation are distinct, $\delta P_{3}=\sum_{n=1}^{8} A_{n} \exp \left(\lambda_{n} x_{3}\right)$ and $\delta S_{11}=$ $\sum_{n=1}^{8} B_{n} \exp \left(\lambda_{n} x_{3}\right)$. Here each coefficient $B_{n}$ can be expressed in terms of $A_{n}$ using Eq. (8), for example; see Ref. [22]. In turn, the coefficients $A_{n}$ must satisfy a system of eight simultaneous equations, which follow from the boundary conditions.
To derive this system, we first employ the continuity of potential $\phi$ and displacement $D_{3}$ at the film-electrode interfaces. Within the film, the perturbation $\delta \phi\left(x_{3}\right)$ can be calculated in terms of $A_{n} \exp \left(\lambda_{n} x_{3}\right)$. The potential $\phi_{m}$ inside electrodes in the presence of a polarization wave becomes $\phi_{m}=\phi_{m 0}\left(x_{3}\right)+\delta \phi_{m}\left(x_{3}\right) \exp \left(i k x_{1}\right)$. The perturbation $\delta \phi_{m}\left(x_{3}\right)$ can be easily found in the screening length approximation to be $\delta \phi_{m}=L_{2} \exp \left(-\zeta x_{3}\right)$, with $\zeta=$ $\sqrt{k^{2}+l_{s}^{-2}}$, in the right electrode $\left(x_{3} \geq 0\right)$ and $\delta \phi_{m}=$ $L_{1} \exp \left[\zeta\left(x_{3}+t\right)\right]$ in the left one $\left(x_{3} \leq-t\right)$. Using these expressions to formulate four boundary conditions and then eliminating $L_{1}$ and $L_{2}$, we obtain $\delta P_{3}=\varepsilon_{0} \varepsilon_{m} \zeta \delta \phi$ at $x_{3}=0$ and $\delta P_{3}=-\varepsilon_{0} \varepsilon_{m} \zeta \delta \phi$ at $x_{3}=-t$. These relationships give us the first two equations for $A_{n}$. Another two equations follow from the conditions imposed on the polarization derivative $P_{3,3}$ [13], which in the approximation of an infinite extrapolation length reduce to $\delta P_{3,3}=0$ at $x_{3}=0$ and $x_{3}=-t$.

The mechanical boundary conditions of the film may be simplified by neglecting the mechanical influence of electrodes in comparison with the substrate effect. (The bottom electrode is assumed to be fully strained by a thick substrate.) Then on the top film surface $\left(x_{3}=0\right)$ the stresses $\sigma_{33}$ and $\sigma_{13}$ may be set to zero. The lattice matching on the bottom film surface implies the continuity of the mechanical displacement and the stresses $\sigma_{33}$ and $\sigma_{13}$ at $x_{3}=-t$. It can be shown that the first of these conditions in our case gives $\delta S_{11}=\delta S_{11}^{\text {sub }}$ and $2 \delta S_{13}-(1 / i k) \delta S_{11,3}=2 \delta S_{13}^{\text {sub }}-$ $(1 / i k) \delta S_{11,3}^{\mathrm{sub}}$ at $x_{3}=-t$. The strains $S_{i j}^{\mathrm{sub}}=\delta S_{i j}^{\mathrm{sub}}\left(x_{3}\right) \times$ $\exp \left(i k x_{1}\right)$ and the stresses $\sigma_{i j}^{\text {sub }}=\delta \sigma_{i j}^{\text {sub }}\left(x_{3}\right) \exp \left(i k x_{1}\right)$ in the nonpiezoelectric cubic substrate can be calculated from the compatibility condition and the equations of mechanical equilibrium. For the function $\delta S_{11}^{\mathrm{sub}}\left(x_{3}\right)$, the calculation gives $\delta S_{11}^{\text {sub }}=R_{1} \exp \left(\beta_{1} k x_{3}\right)+R_{2} \exp \left(\beta_{2} k x_{3}\right)$, where $\beta_{1,2}=\sqrt{\xi \pm \sqrt{\xi^{2}-1}}, \quad \xi=\left(c_{11}^{2}-c_{12}^{2}-4 c_{12} c_{44}\right) /\left(4 c_{11} c_{44}\right)$, and $c_{m n}$ are the elastic stiffnesses of the substrate (set equal to those of the film here). Similar relations can be derived for $\delta S_{13}^{\mathrm{sub}}, \delta S_{33}^{\mathrm{sub}}$, and the functions $\delta \sigma_{i j}^{\mathrm{sub}}\left(x_{3}\right)$ defining the stresses in the substrate [22]. The coefficients $R_{1}$ and $R_{2}$ can be calculated in terms of $A_{n}$ using the strain conditions on the film-substrate interface. The remaining four stress conditions together with the four electrical conditions give us the sought system of eight linear algebraic equations for the coefficients $A_{n}$.

Since this system is homogeneous, a nonzero solution for $A_{n}$ exists only when its determinant $\Delta_{A}$ equals zero. By solving the equation $\Delta_{A}(k)=0$ numerically, it is possible to check the existence of any root $k \neq 0$. If there are no such roots, the uniform polarization state remains stable against inhomogeneous polarization perturbations. The critical thickness $t_{c}$, at which the single-domain state becomes unstable, can be found as a maximum value of $t$ at which a nonzero solution for $k$ first appears.

We performed necessary numerical calculations for fully strained BT and PZT films grown on $\mathrm{SrTiO}_{3}$. Since 


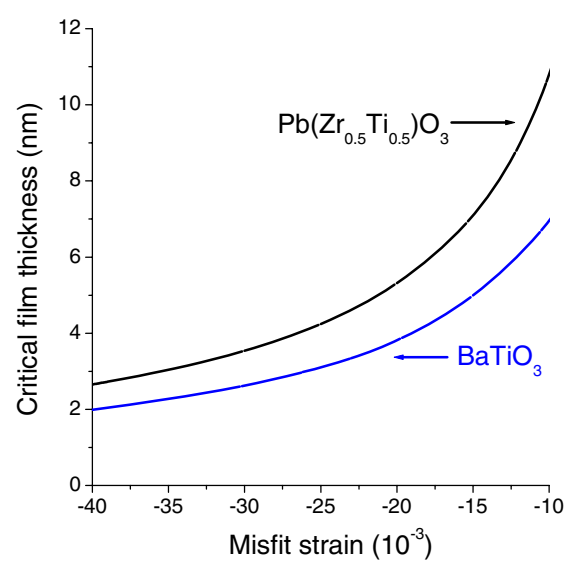

FIG. 2 (color online). Variation of the critical thickness $t_{c, \max }$ with the misfit strain calculated for epitaxial BT and PZT films sandwiched between $\mathrm{SrRuO}_{3}$ electrodes $\left(c_{i}=0.444 \mathrm{~F} / \mathrm{m}^{2}\right)$. The temperature equals $25^{\circ} \mathrm{C}$, and the strain range is restricted to guarantee the stability of the $c$ phase.

for our purposes it is sufficient to determine the upper bound $t_{c, \max }$ of the critical thickness, we simplified the problem by setting the gradient coefficients $g_{l n}$ to zero. The magnitude of $t_{c, \max }$ may be computed for one particular interfacial capacitance $c_{i}$ only, because the critical thickness is inversely proportional to $c_{i}$. We studied the case of two $\mathrm{SrRuO}_{3}$ electrodes $\left(c_{i}=0.444 \mathrm{~F} / \mathrm{m}^{2}\right)$. At room temperature, $t_{c, \max }$ was found to be slightly below $2.98 \mathrm{~nm}$ for BT films and below $2.73 \mathrm{~nm}$ for PZT films. The magnitude of $t_{c, \max }$ weakly decreases with decreasing temperature, reducing at $T=-200^{\circ} \mathrm{C}$ down to about $2.46 \mathrm{~nm}$ in BT films and $2.63 \mathrm{~nm}$ in PZT films.

The calculations also show that $t_{c, \text { max }}$ strongly increases when the magnitude of compressive strain is reduced (see Fig. 2). To clarify the role of the strain effect further, we computed the critical thickness in the absence of electrostrictive coupling ( $q_{l n}$ were set to zero), using the estimates of gradient coefficients $g_{l n}$ given in Ref. [21]. It was found that $t_{c}$ increased up to about $37 \mathrm{~nm}$ for BT films and $56 \mathrm{~nm}$ for PZT films at room temperature. Hence, the strain effect reduces the critical thickness by more than a factor of 10 .

Thus, by combining highly strained epitaxial films with metallic electrodes having good screening properties, it is possible to stabilize the single-domain ferroelectric state in nanoscale capacitors and tunnel junctions. Remarkably, this stabilization results from the elastic effect caused by the electrostrictive coupling between polarization and strain. Our prediction is in line with the recent observation of the monodomain polarization state in ultrathin $\mathrm{PbTiO}_{3}$ films [32]. Moreover, the very small critical thickness calculated for BT films does not contradict the experimental data of Ref. [12], where the 5-nm-thick BT film sandwiched between two $\mathrm{SrRuO}_{3}$ electrodes was found to have relatively large remanent polarization.

The financial support of the Deutsche Forschungsgemeinschaft is gratefully acknowledged.
[1] J. F. Scott, Ferroelectric Memories (Springer, Berlin, 2000).

[2] H. Kohlstedt et al., Phys. Rev. B 72, 125341 (2005).

[3] M. Ye. Zhuravlev et al., Phys. Rev. Lett. 94, 246802 (2005).

[4] E. Y. Tsymbal and H. Kohlstedt, Science 313, 181 (2006).

[5] I. I. Ivanchik, Sov. Phys. Solid State 3, 2705 (1962).

[6] G. M. Guro, I. I. Ivanchik, and N. F. Kovtonyuk, Sov. Phys. Solid State 11, 1574 (1970).

[7] I. P. Batra and B. D. Silverman, Solid State Commun. 11, 291 (1972); I. P. Batra, P. Würfel, and B.D. Silverman, Phys. Rev. Lett. 30, 384 (1973).

[8] R. R. Mehta, B. D. Silverman, and J.T. Jacobs, J. Appl. Phys. 44, 3379 (1973).

[9] D. R. Tilley and B. Žekš, Ferroelectrics 134, 313 (1992).

[10] J. Junquera and Ph. Ghosez, Nature (London) 422, 506 (2003).

[11] C. Lichtensteiger et al., Phys. Rev. Lett. 94, 047603 (2005).

[12] D. J. Kim et al., Phys. Rev. Lett. 95, 237602 (2005).

[13] R. Kretschmer and K. Binder, Phys. Rev. B 20, 1065 (1979).

[14] N. Sai, A. M. Kolpak, and A. M. Rappe, Phys. Rev. B 72, 020101(R) (2005).

[15] G. Gerra et al., Phys. Rev. Lett. 96, 107603 (2006).

[16] T. Mitsui and J. Furuichi, Phys. Rev. 90, 193 (1953); A. Kopal, T. Bahnik, and J. Fousek, Ferroelectrics 223, 127 (1999); A. M. Bratkovsky and A. P. Levanyuk, Phys. Rev. Lett. 84, 3177 (2000); I. Kornev, H. Fu, and L. Bellaiche, Phys. Rev. Lett. 93, 196104 (2004).

[17] E. V. Chenskiǔ and V. V. Tarasenko, Sov. Phys. JETP 56, 618 (1982).

[18] A.M. Bratkovsky and A.P. Levanyuk, arXiv:cond-mat/ 0601484.

[19] N.A. Pertsev, A. G. Zembilgotov, and A. K. Tagantsev, Phys. Rev. Lett. 80, 1988 (1998).

[20] N. A. Pertsev et al., Phys. Rev. B 67, 054107 (2003).

[21] A. G. Zembilgotov et al., J. Appl. Phys. 91, 2247 (2002).

[22] N. A. Pertsev and H. Kohlstedt, arXiv:cond-mat/0603762.

[23] H. Y. Ku and F. G. Ullman, J. Appl. Phys. 35, 265 (1964).

[24] Y.L. Li, L.E. Cross, and L. Q. Chen, J. Appl. Phys. 98, 064101 (2005).

[25] For thermodynamic parameters and material constants used in the calculations, see Ref. [22].

[26] A.L. Roytburd, S. Zhong, and S. P. Alpay, Appl. Phys. Lett. 87, 092902 (2005).

[27] This estimate was obtained using the experimental data of Ref. [12]. The same value follows from the results of firstprinciples calculations [15].

[28] Good agreement of the critical thickness obtained for BT films with the result of the first-principles calculations [10] supports the validity of the Landau theory.

[29] The wave vector $\mathbf{k}$ of polarization perturbation is assumed to be parallel to the [100] crystallographic axis. The case of the [110]-oriented $\mathbf{k}$ can be described in a similar way.

[30] Y. L. Li et al., Appl. Phys. Lett. 78, 3878 (2001).

[31] V. A. Stephanovich, I. A. Luk'yanchuk, and M. G. Karkut, Phys. Rev. Lett. 94, 047601 (2005).

[32] D. D. Fong et al., Phys. Rev. Lett. 96, 127601 (2006); L. Despont et al., Phys. Rev. B 73, 094110 (2006). 